\title{
Coronavirus Disease 2019: One Year Experience at Tertiary Care Cardiac Hospital
}

\author{
Fazila-Tun-Nesa Malik ${ }^{1}$, Md. Kalimuddin ${ }^{3}$, Mir Ishraquzzaman ${ }^{4}$, Mohammad Abdullah Al Mamun ${ }^{7}$, Ashok Dutta ${ }^{2}$, Md. \\ Habibur Rahman ${ }^{2}$, Smita Kanungo ${ }^{5}$, Nazmun Laila ${ }^{5}$, Md. Shamim Chowdhury ${ }^{5}$, Sohel Reza Choudhury ${ }^{6}$
}

\begin{abstract}
:
Background: The Coronavirus Disease 2019 (COVID-19) pandemic is a significant challenge particularly for low and middle-income countries like Bangladesh. Interventions such as home isolation, frequent hand washing, wearing face mask, maintaining social distancing are difficult to implement in densely populated areas. The aim of the study was to delineate demographics, clinical manifestations, treatment modalities and outcomes of COVID-19 affected patients of our hospital.

Methods: This prospective observational study was carried out at National Heart Foundation Hospital \& Research Institute of Bangladesh between 08 March 2020 to 07 March 2021. During this period all admitted patients who subsequently were diagnosed as COVID positive and health care personnel of this hospital, who experienced fever or respiratory symptoms or came in close contact with COVID-19 patients at home or their workplace $\&$ become COVID positive were included.

Results: During this one-year period a total of 769 COVID positive patients were detected in our hospital. Mean age of the patients was $48.16 \pm 15.63$ years (range 1-92 years). Two third were male $(64.9 \%$ vs $35.1 \%)$ and had multiple co-morbidities. One fifth of the patients were $(19 \%)$ asymptomatic. The mean duration of onset of
\end{abstract}

symptoms to test was $3.72 \pm 3.7$ days. Most common symptoms were fever (65.3\%), cough (37.1\%), shortness of breath $(33.6 \%)$ and fatigue $(27.8 \%)$. Other symptoms were bodyache $(18.6 \%)$, headache $(16.6 \%)$, anosmia $16 \%)$, sore throat $(12.1 \%)$, diarrhoea $(6.8 \%)$, dizziness $(5.3 \%)$, generalized itching (3.8\%). Nearly two third of the COVID positive patients $(63.2 \%)$ had a diagnosed cardiovascular disease at onset and remaining $36.8 \%$ patients presented with only COVID-19 disease. About $75 \%$ patients received ivermectin, $5.1 \%$ patients received favipiravir and $4.4 \%$ patients received remdesivir. Three fourth $(\mathbf{7 4 . 3 8 \% )}$ ) of patients were hospitalized and remaining one fourth $(25.62 \%)$ patients were treated either in home isolation or in institutional isolation. Most of the patients recovered, with a case fatality rate of $3.5 \%$. Diabetes, hypertension and age $\geq 50$ years were the independent predictors of mortality.

Conclusion: Although most of the patients had good outcome the study revealed $3.5 \%$ case fatality. Male with multiple co-morbidities were predominantly affected by COVID 19. Fever, cough, shortness of breath and fatigue were common presenting symptoms.

Key words: COVID-19, clinical features, treatment, in-hospital outcome, predictors of mortality

(Bangladesh Heart Journal 2021; 36(2): 89-97)

1. Professor \& Head, Department of Cardiology, National Heart Foundation Hospital \& Research Institute, Mirpur, Dhaka.

2. Associate Professor, Department of Cardiology, National Heart Foundation Hospital \& Research Institute, Mirpur, Dhaka.

3. Assistant Professor, Department of Cardiology, National Heart Foundation Hospital \& Research Institute, Mirpur, Dhaka.

4. Consultant, Department of Cardiology, National Heart Foundation Hospital \& Research Institute, Mirpur, Dhaka.

5. Registrar, Department of Cardiology, National Heart Foundation Hospital \& Research Institute, Mirpur, Dhaka.

6. Professor \& Head, Department of Epidemiology \& Research, National Heart Foundation Hospital \& Research Institute, Mirpur, Dhaka.

7. Assistant Professor, Department of Epidemiology \& Research, National Heart Foundation Hospital \& Research Institute, Mirpur, Dhaka. Address of Correspondence: Prof. Fazila-Tun-Nesa Malik, Professor \& Head, Department of Cardiology, National Heart Foundation Hospital \& Research Institute, Mirpur, Dhaka.

DOI: https://doi.org/10.3329/bhj.v36i2.56034

Copyright () 2017 Bangladesh Cardiac Society. Published by Bangladesh Cardiac Society. This is an Open Access articles published under the Creative Commons Attribution-NonCommercial 4.0 International License (CC BY-NC). This license permits use, distribution and reproduction in any medium, provided the original work is properly cited and is not used for commercial purposes. 


\section{Introduction:}

Like many other countries, Bangladesh has also been experiencing a public health crisis due to Coronavirus Disease 2019 (COVID-19) caused by severe acute respiratory syndrome coronavirus-2 (SARS-CoV-2). As of March 07, 2021, about 117 million confirmed cases and 2.6 million deaths were reported worldwide ${ }^{1}$. The first COVID -19 positive patient was detected in Bangladesh on $8^{\text {th }}$ March 2020. The first case of nonhealth care personnel (non-HCP) COVID-19 was confirmed in our hospital on $14^{\text {th }}$ April and the first case of HCP on $29^{\text {th }}$ April. By $7^{\text {th }}$ March, SARSCoV-2 had caused $5,50,330$ infections and 8,462 deaths in Bangladesh ${ }^{2}$. The common symptoms of the disease include fever, headache, bodyache, shortness of breath, dry cough, sore throat, and fatigue; some patients develop severe pneumonia, acute respiratory distress syndrome, thrombo-embolism, and multiple organ failure progressing to death. However, most affected individuals are healthy asymptomatic carriers or have minor symptoms $^{3}$. SARS-CoV-2 not only involves lung, but may also affect the cardiovascular system ${ }^{4-6}$, gastrointestinal system $^{7,8}$, central nervous system ${ }^{9-11}$, renal system ${ }^{12}$ and $\operatorname{skin}^{13}$. The SARS-CoV-2 protein characteristic spike binds to its cellular receptor, the angiotensin-converting enzyme 2 (ACE2), which is widely expressed in many cell types and organs like lung alveolar cells, nasal epithelium, cerebral cortex, digestive tract, kidney, gallbladder, testis, and adrenal gland ${ }^{11}$. The majority of patients with COVID-19 infection are thought to be paucisymptomatic and do not require hospitalization ${ }^{14}$. Hypertension, diabetes, cardiovascular disease, and pulmonary disease are the most common morbidities among COVID-19 patients ${ }^{15}$. There are many challenges in treatment strategies as there is currently no specific treatment for COVID-19. However, pharmacologic and non-pharmacologic symptom management and supportive care measures should be given to all patients with symptomatic COVID-1915. In spite of its high contagiousness the mortality rate is low. We previously analyzed COVID-19 infection among healthcare personnel ${ }^{16}$. The goal of the present study is to narrate the clinical characteristics, severity of disease at the time of their initial evaluation, treatment and outcome of a large cohort of patients diagnosed with COVID-19 over the initial one year since the first case was declared in Bangladesh.

\section{Methods and Materials:}

Study design, setting, and population

This prospective observational study was carried out in the non-COVID tertiary cardiac care hospital (National
Heart Foundation Hospital \& Research Institute, Dhaka, Bangladesh) from March 08, 2020 to March 07, 2021. All admitted patients, who were subsequently diagnosed as COVID positive and health care personnel of this hospital, who experienced fever or respiratory symptoms or came in close contact with COVID-19 patients at home or their workplace \& later became COVID positive were included in this study. Epidemiological, clinical characteristics, treatment and outcomes data were obtained from data collection forms. The study was approved by the Ethics Review Committee of National Heart Foundation Hospital \& RI (N.H.F.H. \& R.I. 4-14/7/ $A D-1105)$ and written informed consent was obtained from all patients or patient's attendance.

\section{Definition and variables}

A confirmed case of COVID-19 was defined as having a positive result through real-time reverse-transcriptasepolymerase-chain-reaction (RT-PCR) assay of nasopharyngeal swab specimens ${ }^{17}$. We studied confirmed COVID-19 cases only. Demographic information included gender, age, risk factors and co-morbidities (diabetes mellitus, hypertension, smoking, dyslipidemia, obesity, cardiovascular disease, cerebro-vascular disease, chronic obstructive pulmonary disease /bronchial asthma (COPD/BA), chronic kidney disease, pregnancy). The degrees of severity of COVID-19 were classified as mild, moderate, severe, and critical ill ${ }^{18,19}$. Mild type was defined as have mild clinical symptoms without any imaging findings of pneumonia. Moderate type was defined as clinical symptoms (fever or other respiratory symptoms) with imaging findings of pneumonia. Patients with severe type had any of the following parameters: (I) respiratory distress, respiratory rate $\geq 30$ times/min; (II) oxygen saturation $\leq 93 \%$ at rest. Also patients showing a rapid progression $(>50 \%$ ) of chest imaging within $24-$ 48 hours was regarded as severe type. Patients with critical ill type had to meet any of the following standards: (I) respiratory failure requiring mechanical ventilation; (II) shock; (III) complicated extrapulmonary organ failure requiring care in the intensive care unit. The case fatality rate (CFR) was defined as the percentage of the cumulative number of deaths divided by the total number of laboratory-confirmed COVID-19 infections ${ }^{20}$.

Nasopharyngeal swabs collection process

Using a standardized technique, trained doctors or laboratory technicians obtained the nasopharyngeal swabs from patients. After collection, swabs were placed in a transport medium and delivered to the laboratory. Diagnosis of COVID-19 was confirmed by real-time reverse transcription-polymerase chain reaction assay. 


\section{Statistical analysis}

Categorical variables were presented as numbers and percentages and continuous data as mean and standard deviation. Binary logistic regression was used to identify the predictors of mortality. Variables significantly related to in-hospital outcome such as mortality in univariate analysis were included in a binary logistic regression model with the forward method to identify independent predictors of the mortality. A two-sided $p$ value $<0.05$ was considered statistically significant. All analyses were performed using SPSS statistical software version 16.0 (SPSS Inc., Chicago, IL, USA).

\section{Results:}

During one year period a total of 769 patients were infected by SARS-CoV-2. Mean age of the patients was $48.16 \pm 15.63$ years (range 1-92 years), of whom 539 (70.1\%) were non-HCP and 230 (29.9\%) were HCP. Most of the patients were male $(64.9 \%$ vs $35.1 \%)$. Baseline characteristics of COVID-19 patients are shown in Table I.

\section{Table-I}

Baseline characteristics of COVID-19 patients $(n=769)$

\begin{tabular}{|c|c|c|}
\hline Variables & 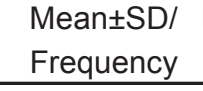 & Percentage \\
\hline Age (Mean $\pm S D)$ in years & $48.16 \pm 15.63$ & \\
\hline \multicolumn{3}{|l|}{ Gender } \\
\hline Male & 499 & 64.9 \\
\hline Female & 270 & 35.1 \\
\hline \multicolumn{3}{|l|}{ Patients category } \\
\hline Non-HCP & 539 & 70.1 \\
\hline $\mathrm{HCP}$ & 230 & 29.9 \\
\hline \multicolumn{3}{|l|}{ Risk factors \& co-morbidities } \\
\hline HTN & 399 & 51.9 \\
\hline $\mathrm{DM}$ & 320 & 41.6 \\
\hline Smoking & 242 & 31.5 \\
\hline Dyslipidemia & 296 & 38.5 \\
\hline Cardiovascular disease & 452 & 58.8 \\
\hline COPD/BA & 54 & 07.0 \\
\hline Obesity & 263 & 34.2 \\
\hline CKD & 247 & 32.1 \\
\hline Pregnancy & 08 & 01.0 \\
\hline \multicolumn{3}{|l|}{ Number of co-morbidities } \\
\hline 0 & 143 & 18.6 \\
\hline 1 & 128 & 16.6 \\
\hline$>1$ & 498 & 64.8 \\
\hline
\end{tabular}

COVID-19: coronavirus disease 2019; HCP: healthcare personnel; non-HCP: non-healthcare personnel; SD: standard deviation; HTN: hypertension; DM: diabetes mellitus; COPD: chronic obstructive pulmonary disease; BA: Bronchial asthma; CKD: chronic kidney disease.
Among the infected, $143(18.6 \%)$ patients did not have any pre-existing comorbidities. Cardiovascular disease $(58.8 \%)$, hypertension $(51.9 \%)$ and diabetes mellitus $(41.6 \%)$ were the most prevalent comorbidities. Other comorbidities are dyslipidemia (38.5\%), obesity $(34.2 \%)$, smoking (31.5\%), chronic kidney disease $(32.1 \%)$ and chronic obstructive pulmonary disease/bronchial asthma $(7 \%)$. Most of the patients had multiple co-morbidities (Figure 1).

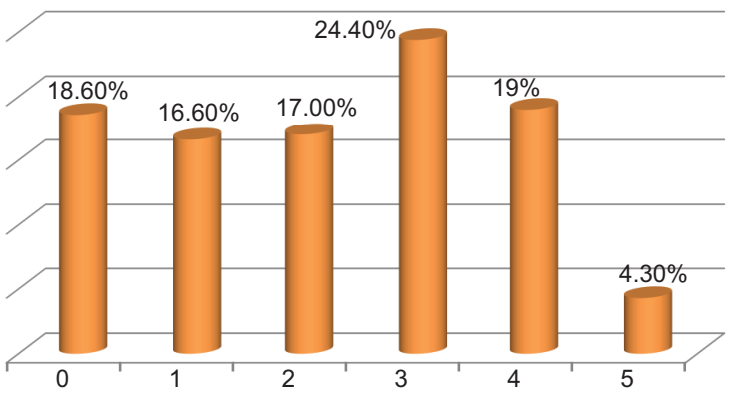

Fig.-1: Bar diagram showing number of co-morbidities of COVID-19 patients ( $n=769)$.

COVID-19: coronavirus disease 2019

It was noted that the infection rate was highest in the month of July during the early stage of the pandemic in Bangladesh. Subsequently, the infection rate plateaued in our hospital (Figure 2).

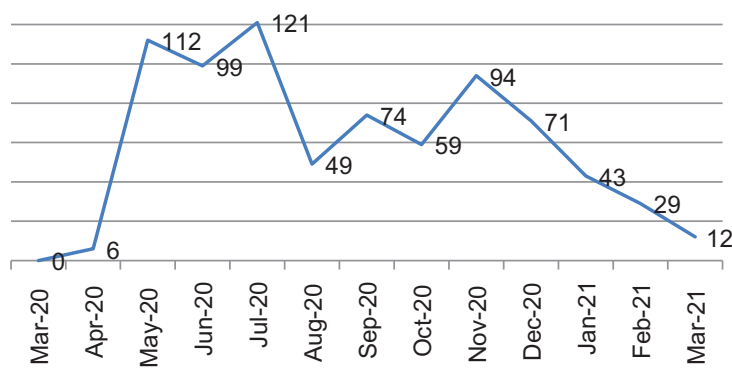

Fig.-2: Bar diagram showing month wise distribution of COVID-19 patients $(n=769)$

COVID-19: coronavirus disease 2019

About $81 \%$ of the patients were symptomatic and $19 \%$ patients were asymptomatic. The mean duration of onset of symptoms to test was $3.72 \pm 3.7$ days. Patients had clinical manifestations of fever (502 [65.3\%] patients), cough (285 [37.1\%] patients), shortness of breath (258 [33.6\%] patients), fatigue (214 [27.8\%] patients), bodyache (143 [18.6\%] patients), headache (128 [16.6\%] patients), anosmia (123 [16\%] patients), 
sore throat (93 [12.1\%] patients), diarrhoea (52 [6.8\%] patients), dizziness (41 [5.3\%] patients), generalized itching (29 [3.8\%] patients), vomiting (27 [3.5\%] patients) nausea (20 [2.6\%] patients), anorexia (15 [2.0\%] patients), abdominal pain (10 [1.3\%] patients) and numbness (10 [1.3\%] patients). Clinical characteristics of 769 confirmed COVID-19 patients are outlined in Table II. Most of the patients had good left ventricular function (64\%).

Table-II

Clinical characteristics of COVID-19 positive patients $(n=769)$

\begin{tabular}{lcc}
\hline Variables & Frequency & Percentage \\
\hline Clinical presentation & & \\
Symptomatic & 630 & 81.1 \\
Asymptomatic & 139 & 18.9 \\
Presenting symptoms & & \\
Fever & 502 & 65.3 \\
Fatigue & 214 & 27.8 \\
Cough & 285 & 37.1 \\
Bodyache & 143 & 18.6 \\
Headache & 128 & 16.6 \\
Anosmia & 123 & 16.0 \\
Sore throat & 93 & 12.1 \\
Shortness of breath & 258 & 33.6 \\
Diarrhea & 52 & 6.8 \\
Dizziness & 41 & 5.3 \\
Generalized itching & 29 & 3.8 \\
Vomiting & 27 & 3.5 \\
Nausea & 20 & 2.6 \\
Anorexia & 15 & 2.0 \\
Abdominal pain & 10 & 1.3 \\
Numbness & 10 & 1.3 \\
Left ventricular ejection fraction & & \\
Good & 492 & 64.0 \\
Mild & 165 & 21.4 \\
Moderate & 92 & 12.0 \\
Severe & 20 & 2.6 \\
\hline COVID-19: & &
\end{tabular}

COVID-19: coronavirus disease 2019.

Most of the patients (63.2\%) had a cardiac diagnosis: Acute coronary syndrome [ST-segment elevation myocardial infarction (13.4\%); non-ST segment elevation myocardial infarction (10.6) \& unstable angina (7.9\%)]; chronic coronary syndrome $(22.8 \%)$; ischemic cardiomyopathy $(2.1 \%)$; valvular heart disease $(3.6 \%)$; sinus \& atrio-ventricular node disease $(1.1 \%)$, congenital heart disease $(1.3 \%)$ and dilated cardiomyopathy $(0.4 \%)$. Remaining $36.8 \%$ patients had only COVID-19 disease.
All patients were treated in isolation. Oxygen therapy (low flow, high flow) was given when required. Prone positioning was advised for all patients. Treatment outline is given in Table III. Most of the patients received ivermectin (576 [74.9\%]). Only four $(0.5 \%)$ patients received hydroxychloroquine at the early period of COVID-era. Total $39(5.1 \%)$ patients received favipiravir (1600 mg on day 1 followed by $600 \mathrm{mg} 12$ hourly from day 2 to day 10) and $34(4.4 \%)$ patients received remdesivir (200 mg IV infusion [within $30 \mathrm{~min}-2$ hours] on day 1 followed by $100 \mathrm{mg}$ infusion within [30 min to 2 hours] from day 2 to day 5). One $(0.1 \%)$ patient was also treated by 2 doses of tocilizumab ( $8 \mathrm{mg} / \mathrm{kg}$ [max: $800 \mathrm{mg} / \mathrm{dose}$ ]) and 1 dose of convalescent plasma therapy due to cytokine storm.

Table-III

Distribution of treatment of COVID-19 patients $(n=769)$

\begin{tabular}{lcc}
\hline Variables & Frequency & Percentage \\
\hline Antibiotics & & \\
$\quad$ IV & 123 & 16.0 \\
Oral + IV & 65 & 8.4 \\
$\quad$ Oral & 498 & 64.8 \\
$\quad$ Not received & 83 & 10.8 \\
Antibiotics & & \\
$\quad$ Single & 516 & 67.1 \\
$\quad$ Double & 170 & 22.1 \\
$\quad$ Not received & 83 & 10.8 \\
Steroids (oral \& IV) & 56 & 7.3 \\
Favipiravir & 39 & 5.1 \\
Remdesivir & 34 & 4.4 \\
Ivermectin & 576 & 74.9 \\
Hydroxy-chloroquine & 4 & 0.5 \\
Enoxaparine & 483 & 62.8 \\
Rivaroxaban & 485 & 63.1 \\
Tocilizumab & 1 & 0.1 \\
\hline
\end{tabular}

COVID-19: coronavirus disease 2019; IV: intravenous.

Regarding antibiotic therapy, 516 (67.1\%) patients were treated with a single antibiotic and $170(22.1 \%)$ patients were given combination therapy. Rest of the patients $(10.8 \%)$ did not require antibiotic therapy. The antibiotics used generally covered common pathogens. The antibiotics used were doxycycline, macrolide, cephalosporins, fluoroquinolones, carbapenems and $\beta$ lactamase inhibitors. Regarding oral antibiotic treatment, $275(35.8 \%)$ patients were treated by azithromycin and $341(44.3 \%)$ patients by doxycycline. Most of the patients received either ivermectin plus azithromycin or ivermectin plus doxycycline combination. The duration of antibiotic treatment was 5-10 days. Around 56 (7.3\%) patients were also treated with methylprednisolone and 
dexamethasone for 3-7 days. Low molecular weight heparin was used in $483(62.8 \%)$ patients and newer oral anticoagulant (rivaroxaban $10 \mathrm{mg}$ once daily for 1 month) was used in $485(63.1 \%)$ patients. We administered vitamin $C$, vitamin $D_{3}$ and zinc to most of the patients.

Total $572(74.38 \%)$ patients were hospitalized and remaining 197 (25.62\%) patients were treated either in home isolation or in institutional isolation. Regarding disease severity, $18.1 \%$ patients had asymptomatic disease, $67.3 \%$ had mild disease; $6.6 \%$ had moderate disease; $6.2 \%$ had severe disease and $1.8 \%$ were critically ill. Case fatality rate (CFR) was $3.5 \%$ (Table IV).

Table-IV

In-Hospital outcome of COVID-19 patients (769)

\begin{tabular}{lcc}
\hline Variables & Frequency & Percentage \\
\hline Hospitalization & 572 & 74.38 \\
Home isolation & 197 & 25.62 \\
Disease severity & & \\
Asymptomatic & 139 & 18.1 \\
Mild & 517 & 67.3 \\
Moderate & 51 & 6.6 \\
Severe & 48 & 6.2 \\
Critical ill & 14 & 1.8 \\
Mortality & 271 & 3.5 \\
\hline
\end{tabular}

COVID-19: coronavirus disease 2019.

Table $V$ shows the univariate analysis of in-hospital outcome of study population. Age more than 50 years, non-health care personnel, presence of cardiovascular disease, Left ventricular ejection fraction (LVEF) category, disease severity and diabetes mellitus significantly related with in-hospital mortality.

Among 769 patients, 27 (3.5\%) patients died. Univariate analysis showed several factors were significantly related with in-hospital mortality. Based on these variables, binary logistic regression using the forward method was performed, and we found that diabetes mellitus, hypertension and age more than 50 years were the independent predictor of mortality (Table VI).
Table-V

Distribution of factors associated with in hospital outcome of study population ( $n=769$ )

\begin{tabular}{|c|c|c|c|}
\hline \multirow[t]{2}{*}{ Variables } & \multicolumn{2}{|c|}{ Outcome } & \multirow[t]{2}{*}{$\mathrm{P}$ value } \\
\hline & $\begin{array}{l}\text { In hospital } \\
\text { deathf }(\%)^{\#}\end{array}$ & $\begin{array}{c}\text { Recovered } \\
\mathrm{f}(\%)^{\#}\end{array}$ & \\
\hline \multicolumn{4}{|l|}{ Age group } \\
\hline$<50 \mathrm{Y}$ & $04(14.8)$ & $364(49.1)$ & 0.000 \\
\hline$>50 \mathrm{Y}$ & $23(85.2)$ & $378(50.9)$ & \\
\hline \multicolumn{4}{|l|}{ Gender } \\
\hline Male & $20(74.1)$ & $479(64.6)$ & 0.210 \\
\hline Female & $07(25.9)$ & $263(35.4)$ & \\
\hline \multicolumn{4}{|l|}{ Non-HCP/HCP } \\
\hline Non-HCP & $26(96.3)$ & $513(69.1)$ & 0.001 \\
\hline $\mathrm{HCP}$ & $01(03.7)$ & $229(30.1)$ & \\
\hline \multicolumn{4}{|l|}{ CVD } \\
\hline Present & $21(10.2)$ & $311(41.9)$ & 0.030 \\
\hline Absent & $06(0.6)$ & $431(58.1)$ & \\
\hline \multicolumn{4}{|l|}{ Obesity } \\
\hline Non obese & $19(70.4)$ & $487(65.6)$ & 0.388 \\
\hline Obese & $08(29.6)$ & $255(34.4)$ & \\
\hline \multicolumn{4}{|l|}{ LVEF Category } \\
\hline Severe LV Dysfunction & $03(11.1)$ & $17(02.3)$ & 0.007 \\
\hline Moderate LV Dysfunction & $05(18.5)$ & $87(11.7)$ & \\
\hline Mild LV Dysfunction & $08(29.6)$ & $157(21.2)$ & \\
\hline Good Function & $11(40.7)$ & $481(64.8)$ & \\
\hline \multicolumn{4}{|l|}{ Disease Severity } \\
\hline Asymptomatic & $01(03.7)$ & $138(18.6)$ & 0.000 \\
\hline Mild & $03(11.1)$ & $514(69.3)$ & \\
\hline Moderate & $01(03.7)$ & $50(06.7)$ & \\
\hline Severe & $13(48.1)$ & $35(04.7)$ & \\
\hline Critically ill & $09(33.3)$ & $05(0.70)$ & \\
\hline \multicolumn{4}{|l|}{ Diabetes Mellitus } \\
\hline Diabetic & $20(74.1)$ & $300(40.4)$ & 0.001 \\
\hline Non diabetic & 07 (25.9) & $442(59.6)$ & \\
\hline \multicolumn{4}{|l|}{ Blood Pressure } \\
\hline Hypertensive & $13(48.1)$ & $386(52.0)$ & 0.420 \\
\hline Normotensive & $14(51.9)$ & $356(48.0)$ & \\
\hline
\end{tabular}

HCP: healthcare personnel; non-HCP: non-healthcare personnel; CVD: cardiovascular disease; LVEF: left ventricular ejection fraction. \# Value in the parenthesis shows the corresponding row percentage; *Chi square test to find out significance. 
Table-VI

Multivariate analysis of in hospital outcome of study population ( $n=769)$

\begin{tabular}{lccccc}
\hline & Wald & Sig. & Exp(B) & \multicolumn{2}{c}{$95.0 \%$ C.I. for Exp(B) } \\
\cline { 5 - 6 } & & & & Lower & Upper \\
\hline Age $\geq 50$ years & 4.457 & 0.035 & 0.281 & 0.086 & 0.913 \\
Gender Male & 0.104 & 0.747 & 1.167 & 0.456 & 2.986 \\
Non-HCP/HCP & 3.705 & 0.054 & 9.411 & 0.960 & 92.260 \\
CVD & 1.964 & 0.161 & 2.206 & 0.730 & 6.668 \\
Obese & 0.365 & 0.546 & 1.311 & 0.545 & 3.155 \\
LV Dysfunction & 0.634 & 0.426 & 0.702 & 0.294 & 1.676 \\
Disease Severity & 3.670 & 0.055 & 0.136 & 0.018 & 1.047 \\
DM & 5.861 & 0.015 & 0.314 & 0.123 & 0.802 \\
HTN & 7.147 & 0.008 & 3.112 & 1.354 & 7.153 \\
Constant & 0.777 & 0.378 & 8.074 & & \\
\hline
\end{tabular}

a. Variable(s) entered on step 1: Age $\geq 50$ years, Gender (Male), NOHCP/HCP, CVD, Obese, LV Dysfunction, Disease severity, DM, HTN.

HCP: healthcare personnel; non-HCP: non-healthcare personnel; CVD: cardiovascular disease; LV: left ventricular; DM: diabetes mellitus; HTN: hypertension; Sig.: significant; C.I.: confidence interval; Exp: exponential.

\section{Discussion:}

Important findings of this study are: 1) Most of the patients were male; 2) Most of the patients had multiple comorbidities; 3) Around 19\% patients had asymptomatic presentation; 4) Most common symptoms of COVID-19 were fever, cough \& shortness of breath; and 5) Most of the patients had mild disease with a low mortality rate (3.5\%).

Our study was based in a large tertiary cardiac care hospital in Dhaka, Bangladesh. Many COVID-19 affected patients present with cardiovascular symptoms. Also, many cardiac patients have underlying COVID-19 infection. Our hospital is a non-COVID hospital. However, during the pandemic many patients were admitted who were subsequently diagnosed with COVID-19. Undoubtedly taking care of these non-HCP put the HCP of our hospital at risk of becoming infected with COVID-19.

Analysis of 207,079 RT-PCR positive patients in Argentina showed mean age of the patient $42.9 \pm 18.8$ years and $50.0 \%$ were males ${ }^{17}$. Most common symptoms were fever, cough, headache and sore throat. Death or intensive care unit admission were independently associated with older age, male, coma, dyspnea or tachypnea, and seizures, with underlying co-morbidities such as immunodeficiency, chronic renal failure, and liver disease showing the strongest effects. The burden of SARS-CoV-
2 infection among healthcare personnel was $10.6 \%$, The total case fatality rate was $5.3 \%$.

Meta-analysis of sixty studies included a total of 59,254 patients from 11 countries $^{21}$ and detected the most common symptoms in patients with SARS-CoV-19 infection were fever, cough, muscle aches and/or fatigue and dyspnea. Overall, the male/female ratio was 1.08. All-cause mortality was $0.3 \%$. Epidemiological studies showed that mortality was higher in males and elderly patients.

Summary of a report of 72314 cases from the Chinese Center for Disease Control and Prevention ${ }^{22}$ showed among a total of 72314 case records, 44672 were classified as confirmed cases of COVID-19 (62\%) and asymptomatic cases (1\%). Most case patients were 30 to 79 years of age $(87 \%)$. Most cases were classified as mild $(81 \%)$. However, $14 \%$ were severe and $5 \%$ were critical. The overall case-fatality rate (CFR) was $2.3 \%$. Infection rate among health care personnel were $3.8 \%$.

Result from five retrospective clinical studies ${ }^{3}$, which included a total of 1556 hospitalized patients with COVID19 and showed $57.5 \%$ were male and mean age of the patients 49.1 years. Common symptoms were fever, cough and fatigue. Critical cases with complications were $9 \%$, intensive care unit admission was required in $7.3 \%$, invasive ventilation in $3.4 \%$, and mortality was $2.4 \%$. 
Clinical and epidemiological characteristics of 1420 European patients with mild-to-moderate coronavirus disease $2019^{14}$ showed $67.7 \%$ were females and $30.7 \%$ patients were HCP. The mean age of patients was $39.17 \pm 12.09$ years. The most common symptoms were headache, loss of smell, nasal obstruction, cough, asthenia, myalgia, rhinorrhea, gustatory dysfunction and sore throat. Fever was reported by $45.4 \%$. The prevalence of symptoms significantly varied according to age and sex. Young patients more frequently had ear, nose and throat complaints, whereas elderly individuals often presented fever, fatigue and loss of appetite. Loss of smell, headache, nasal obstruction and fatigue were more prevalent in female patients.

Analysis of eighty articles ${ }^{15}$ included a total of 417 patients with a mean age of 48 years. The most common clinical manifestations were fever, cough, dyspnea, and myalgia or fatigue. Less common clinical manifestations included nausea or vomiting, dizziness, rhinorrhea, and chills. Hypertension, diabetes, cardiovascular disease, and pulmonary disease were the most common morbidities among COVID-19 patients.

Analysis of 140 hospitalized COVID-19 patients in Wuhan $^{23}$ revealed $50.7 \%$ of patients were male with an overall median age of 57.0 years. Fever, cough and fatigue were the most common symptoms in COVID-19 patients. More than $1 / 3$ of the patients had chest tightness or dyspnea and gastrointestinal symptoms such as nausea, diarrhea, and anorexia. Hypertension and diabetes mellitus were the most common comorbidities.

Retrospective, single-center case series of the 138 hospitalized patients with confirmed novel coronavirusinfected pneumonia in Wuhan, China showed ${ }^{24}$ the median age of the patients was 56 years. Common symptoms were fever, fatigue and dry cough. The overall mortality rate was $4.3 \%$.

A cohort study ${ }^{25}$ on 201 Bangladeshi patients was done in Combined Military Hospital, a tertiary level hospital in Dhaka, Bangladesh from April 2020 to May 2020. Mean age of the patients was $32.2 \pm 2$ years and $90 \%$ were male. Common symptoms are fever, cough, headache, myalgia, sore throat, malaise, respiratory distress. Asymptomatic cases were $4.5 \%$. Death rate was $1 \%$ which was associated with comorbidity of CKD.

Mean age of our study population was 48 years, which is consistent with other studies ${ }^{3,15}$. However, in some studies $^{14,17,25}$ mean age of the patients was less than our study and some other studies, median age was greater than our studies (56-57 years $)^{23,24}$. Male were predominant in our study as also in other studies $3,21,23,25$.

The COVID-19 pandemic has shown a striking gender bias with more cases and a higher mortality rate in men than in women ${ }^{26}$. Increased male susceptibility might be explained by biological and behavioral factors. Biological factors include men's high level of testosterone that inhibits antibody production, and the presence of angiotensin-converting enzyme 2 (ACE2) receptors (cell receptors which play an essential role in SARS-CoV-2 entry) that facilitate viral replication ${ }^{27}$. Similarly, behavioral and lifestyle factors include men's higher rates of smoking and low level of hand-washing practices [28], although there is no clear evidence these behavioral factors have any impact on COVID-19 transmission.

Most common symptoms in our study were fever, cough, shortness of breath and fatigue which are almost consistent with other studies $3,15,17,21-24$. In contrast to our study, one European study ${ }^{14}$ showed female predominance and the most common symptoms were headache, loss of smell, nasal obstruction, cough, asthenia, myalgia, rhinorrhea, gustatory dysfunction and sore throat.

As COVID-19 involves multisystem, it also affects olfactory and gastrointestinal tract (GIT). In our study, 16\% patients developed anosmia. In one of the first studies from China ${ }^{10}$, anosmia was mentioned to affect only about $5.1 \%$ of COVID-19 patients. Another study from France reported $47 \%$ patients with confirmed COVID-19 had anosmia ${ }^{11}$. The pathophysiological mechanism underlying the occurrence of anosmia is still not wellunderstood, but two explanations have been proposed ${ }^{12}$. The first hypothesis suggests peripheral viral involvement. Another hypothesis, currently the most widely accepted, suggests the direct changes to the central nervous system by the virus ${ }^{12}$. SARS-CoV-2 infection is strongly associated with the development of anosmia , especially in females and those with fever ${ }^{12}$.

In our study, diarrhea (6.8\%) was the most common GIT symptom. Other symptoms were nausea, vomiting, altered taste and abdominal pain. SARS-CoV-2 infects the GI tract via its viral receptor angiotensin converting enzyme II, which is expressed on enterocytes of the ileum and colon ${ }^{7}$. Viral ribonucleic acid (RNA) has also been isolated from stool specimens of COVID-19 patients, which raised the concern for fecal-oral transmission in addition to droplet transmission ${ }^{7}$.

The largest cases of patients (74 patients) with COVID19 with Gl symptoms outside Wuhan showed its novel characteristics of increased family clustering and liver 
injury, severe/critical tendency and higher rate of body temperature $>38.5^{\circ} \mathrm{C}$ [8]. Among enrolled 651 patients, $74(11.4 \%)$ presented with at least one gastro-intestinal symptom (nausea, vomiting or diarrhoea) ${ }^{8}$.

In our study, most cases were classified as mild which is consistent with other studies $3,14,21,22,25$. A total of 8096 severe acute respiratory syndrome (SARS) cases and 774 deaths across 29 countries were reported for an overall CFR of $9.6 \% 22$. Middle East respiratory syndrome (MERS) is responsible for 2494 confirmed cases and 858 deaths across 27 countries for a CFR of $34.4 \% 22$. Despite much higher CFRs for SARS and MERS, COVID19 has led to more total deaths due to the large number of cases. In our study, case fatality rate was $3.5 \%$ which is comparable with other studies $3,22,25$.

Asymptomatic infection is often understood as detection of SARS-CoV-2 by RT-PCR, in the absence of a clinical illness compatible with COVID-1929. Asymptomatic transfer leads to lower prevalence estimates and higher transmission rates in the community. Various reports worldwide showed various COVID-19 asymptomatic case rates diverse from $1.2 \%$ to $51.4 \%{ }^{30}$. In our study, $18.9 \%$ patients had asymptomatic infection. Public health measures, including quarantining in the community, frequent hand washing, maintaining social distancing, wearing mask, to trace close contacts of those testing positive for COVID-19 as well as timely diagnosis and strict adherence to universal precautions in health care settings, are critical in controlling COVID-19.

Our study has some limitations: First, it is a single centre study. So, it may not reflect the true scenario of the entire country. Second, it is non-COVID dedicated hospital. Only patients with cardiac symptoms were admitted. Third, cardiac manifestations of COVID-19 could not be evaluated as almost all non-HCP were cardiac patients.

\section{Conclusion:}

Regarding prevalence of COVID 19

Male were predominant with multiple co-morbidities. Most common symptoms were fever, cough, shortness of breath and fatigue. Most patients had good outcome. Asymptomatic cases of SARS-CoV-2 can be unknown carriers magnifying the transmission of COVID-19. Thus, mask wearing, maintaining social distance, extensive testing for identification and the quarantine of infected asymptomatic individuals are essential to curb this pandemic. Diabetes, hypertension and age $\geq 50$ years were the independent predictors of mortality.

\section{Acknowledgement:}

We would like to thank Mrs Rehana Akter and Mr. Rasel Hasan, National Heart Foundation Hospital \& Research Institute, for their sincere hard work in collecting and tabulating the data.

\section{References:}

1. Worldometer: Corovirus update, World data. (2021). Accessed: March 7, 2021: https://www.worldometers. info/coronavirus/.

2. Worldometer: Corovirus update, Bangladesh data. (2021). Accessed: March 7, 2021: https:// www.worldometers.info/coronavirus/ country/ bangladesh/.

3. Lovato A, de Filippis C: Clinical presentation of COVID-19: a systematic review focusing on upper airway symptoms. Ear Nose Throat J. 2020;99:569576. 10.1177/0145561320920762

4. Efros O, Barda N, Meisel E, et al.: Myocardial injury in hospitalized patients with COVID-19 infectionRisk factors and outcomes. PLoS ONE. 2021;16: e0247800. 10.1371/ journal.pone.0247800

5. Chang W-T, Toh HS, Liao C-T, Yu W-L: Cardiac Involvement of COVID-19: A Comprehensive Review. Am J Med Sci. 2021;361:14-22. 10.1016/j.amjms. 2020.10.002

6. Pericas JM, Hernandez-Meneses M, Sheahan TP, et al.: COVID-19: from epidemiology to treatment. Eur Heart J. 2020;41:2092-2108. 10.1093/ eurheartj/ehaa462

7. Cha MH, Regueiro M, Sandhu DS. Gastrointestinal and hepatic manifestations of COVID-19: A comprehensive review. World J Gastroenterol. 2020;26:2323-2332. 10.3748/wjg.v26.i19.2323

8. Jin X, Lian J-S, Hu J-H, et al.: Epidemiological, clinical and virological characteristics of 74 cases of coronavirus-infected disease 2019 (COVID-19) with gastrointestinal symptoms. Gut. 2020;69:10021009. 10.1136/gutjnl-2020-320926

9. Mao L, Jin $H$, Wang $M$, et al.: Neurologic manifestations of hospitalized patients with coronavirus disease 2019 in Wuhan, China. JAMA Neurol. 2020;77:683-690. 10.1001/jamaneurol.2020. 1127

10. Klopfenstein T, Kadiane-Oussou NJ, Toko L, Royer PY, Lepiller Q, Gendrin V, Zayet S: Features of anosmia in COVID-19. Med Mal Infect. 2020;50:436439. 10.1016/j.medmal.2020.04.006 
11. da Silva PR Jr, Gomes ALOR, Coelho LEA, et al.: Anosmia and COVID-19: perspectives on its association and the pathophysiological mechanisms involved. The Egyptian Journal of Neurology, Psychiatry and Neurosurgery. 2021;57:18. 10.1186/s41983-020-00266-0

12. Migliaccio MG, Di Mauro M, Ricciolino R, et al.: Renal Involvement in COVID-19: A Review of the Literature. Infection and Drug Resistance. 2021;14:895-803. 10.2147/IDR.S288869

13. Almutairi A, Alfaleh $M$, Alasheikh M: Dermatological Manifestations in Patients with SARSCoV-2: A Systematic Review. Cureus. 2020;12(7): e9446. $10.7759 /$ cureus. 9446

14. Lechien JR, Chiesa Estomba CM, Place S, et al.: Clinical and epidemiological characteristics of 1420 European patients with mild to moderate coronavirus disease 2019. JIM. 2020;288:335-344. 10.1111/joim.13089

15. Tahvildari A, Arbabi M, Farsi Y, et al.: Clinical Features, Diagnosis, and Treatment of COVID-19 in Hospitalized Patients: A Systematic Review of Case Reports and Case Series. Front. Med. 2020;7:231. 10.3389/fmed.2020.00231

16. Malik F, Ishraquzzaman M, Kalimuddin M, et al.: Clinical Presentation, Management and In-Hospital Outcome of Healthcare Personnel With COVID-19 Disease. Cureus. 2020;12(8): e10004. 10.7759/ cureus. 10004

17. Schonfeld D, Arias S, Bossio JC, Fernandez H, Gozal D, Perez-Chada D: Clinical presentation and outcomes of the first patients with COVID-19 in Argentina: Results of 207079 cases from a national database. PLoS ONE. 2021;16: e0246793. 10.1371/journal.pone.0246793

18. Zu ZY, Jiang MD, Xu PP, Chen W, Ni QQ, Lu GM, Zhang LJ: Coronavirus Disease 2019 (COVID-19): A Perspective from China. Radiology. 2020;296:E15-25. 10.1148/radiol.2020200490

19. Yue H, Bai X, Wang J, et al.: Clinical characteristics of coronavirus disease 2019 in Gansu province, China. Ann Palliat Med. 2020;9:1404-1412. 10.21037/apm-20-887

20. Zheng L, Wang $X$, Zhou $C$, et al.: Analysis of the infection status of the health care workers in Wuhan during the COVID-19 outbreak: A cross-sectional study. Clin Infect Dis. 2020;71:2109-2113. 10.1093/ $\mathrm{cid} / \mathrm{ciaa} 588$
21. do Nascimento IB jr, Cacic N, Abdulazeem HM, et al.: Novel Coronavirus Infection (COVID-19) in Humans: A Scoping Review and Meta-Analysis. J. Clin. Med. 2020;9: 941. 10.3390/jcm9040941

22. Wu Z, McGoogan JM: Characteristics of and important lessons from the coronavirus disease 2019 (COVID-19) outbreak in China: summary of a report of 72314 cases from the Chinese Center for Disease Control and Prevention. JAMA. 2020;323:1239-1242. 10.1001/jama.2020.2648

23. Zhang J-J, Dong X, Cao Y-Y, et al.: Clinical characteristics of 140 patients infected with SARSCoV-2 in Wuhan, China. Allergy. 2020;75:1730-1741. 10.1111/all.14238

24. Wang D, Hu B, Hu C, et al.: Clinical characteristics of 138 hospitalized patients with 2019 novel coronavirus-infected pneumonia in Wuhan, China. JAMA. 2020;323:1061-1069. 10.1001/ jama.2020.1585

25. Ahmed NU, Islam MA, Kabir MA, Rahman $\mathrm{MH}$, Sadat SMA: Clinico-Pathological Findings of Bangladeshi Covid 19 Patients with their Clinical Outcome: Study of A Cohort of 201 Cases. J Bangladesh Coll Phys Surg. 2020;38:37-42. 10.3329/jbcps.v38i0.47346

26. Gadi N, Wu SC, Spihlman AP, MoultonVR: What's sex got to do with COVID-19? Gender-based differences in the host immune response to Coronaviruses. Front Immunol. 2020;11:2147. 10.3389/fimmu.2020.02147

27. Cao Y, Li L, Feng Z, et al.: Comparative genetic analysis of the novel ACE2 in different populations. Cell Discov. 2020;6:11. 10.1038/s41421-020-01471

28. Acharya Y, Pant S, Gyanwali P, Dangal G, Karki P, Bista NR, Tandan M: Gender disaggregation in COVID-19 and increased male susceptibility. J Nepal Health Res Counc. 2020;18:345350. 10.33314/jnhrc.v18i3.3108

29. Keeley AJ, Evans CM, de Silva TI: Asymptomatic SARS-CoV-2 infection: the tip or the iceberg? Thorax. 2020;75:621-622. 10.1136/thoraxjnl-2020215337

30. Rachman BE, Rusli M, Miftahussurur M: The Hidden Vulnerability of COVID-19 Observed From Asymptomatic Cases in Indonesia. Sys Rev Pharm. 2020;11:703-713. 10.31838/srp.2020.2.103 